International Journal of Pure and Applied Mathematics

Volume 117 No. 1 2017, 247-254

ISSN: 1311-8080 (printed version); ISSN: 1314-3395 (on-line version)

url: http://www.ijpam.eu

doi: $10.12732 /$ ijpam.v117i1.18

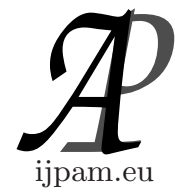

\title{
ANOTHER STEP TOWARDS DETERMINING THE HIT NUMBER OF SUBDIVIDED STARS
}

\author{
Jozef Bucko ${ }^{1}$, Július Czap ${ }^{2} \S$ \\ ${ }^{1,2}$ Department of Applied Mathematics and Business Informatics \\ Faculty of Economics \\ Technical University of Košice \\ Němcovej 32, 04001 Košice, SLOVAKIA
}

\begin{abstract}
A total-coloring $c$ of a directed graph $G$ is called edge-distinguishing if for any two edges $e_{1}=u_{1} v_{1}$ and $e_{2}=u_{2} v_{2}$ of $G$ the associated ordered triplets $\left(c\left(u_{1}\right), c\left(e_{1}\right), c\left(v_{1}\right)\right)$ and $\left(c\left(u_{2}\right), c\left(e_{2}\right), c\left(v_{2}\right)\right)$ are different. The problem is to determine the minimum number of colors used in such a coloring of $G$. In this paper we investigate this parameter for directed subdivided stars.
\end{abstract}

AMS Subject Classification: 05C15, 05C20, 05C 78

Key Words: directed graph, total-coloring

\section{Introduction}

An edge-labeling of a graph $G$ is an assignment of labels to the edges of $G$, one label to each edge. An edge-labeling of $G$ is edge-distinguishing if no two edges of $G$ are assigned the same label. That is, each edge of $G$ is uniquely determined by its label. Edge-distinguishing labelings can be defined in different ways.

A graceful labeling $f$ of a connected graph $G$ with $m$ edges is a labeling of its vertices with labels $0,1, \ldots, m$ such that no two vertices have the same label, and the induced edge-labeling, which labels the edge $u v$ with integer

Received:

Revised:

2017-10-18

2017-11-13

Published: December 12, 2017

${ }^{\S}$ Correspondence author (c) 2017 Academic Publications, Ltd. url: www.acadpubl.eu 
$|f(u)-f(v)|$, assigns distinct labels to the edges of $G$. Thus the set of labels of the edges of $G$ in every graceful labeling is $\{1,2, \ldots, m\}$. Hence every graceful labeling is edge-distinguishing. Graceful labelings of graphs were introduced by Rosa [10], although the term "graceful" was first used by Golomb [6]. A graph that admits a graceful labeling is a graceful graph. Note that not all graphs are graceful. A major problem in this area is that of determining which graphs are graceful. The most famous conjecture in this area is the following.

Conjecture 1. Every tree is graceful.

13 years after Rosa introduced graceful labeling, Graham and Sloane [7] introduced a harmonious labeling. A harmonious labeling of a connected graph $G$ with $m$ edges is an assignment $f$ of distinct elements of the set $\mathbb{Z}_{m}=$ $\{0,1, \ldots, m-1\}$ to the vertices of $G$ so that the resulting edge-labeling in which each edge $u v$ is labeled with $f(u)+f(v)$ (addition modulo $m$ ) is edgedistinguishing. Since such a vertex-labeling is not possible if $G$ is a tree, in this case, some element of $\mathbb{Z}_{m}$ is assigned to two vertices of $G$, while all other elements of $\mathbb{Z}_{m}$ are used exactly once. A graph that admits a harmonious labeling is called a harmonious graph. While many classes of trees have been shown to be harmonious, it is not known whether all trees are harmonious. Graham and Sloane made the following conjecture, which parallels that of a famous conjecture on graceful labelings of trees.

Conjecture 2. Every tree is harmonious.

A harmonious coloring of a graph $G$ is a proper vertex-coloring of $G$ having the property that if $i$ and $j$ are two distinct colors used in the coloring of $G$, then there is at most one pair of adjacent vertices assigned these two colors. Evidently, every harmonious coloring $c$ of $G$ induces an edge-labeling of $G$ where the edge $u v$ is assigned the label $\{c(u), c(v)\}$, which is then a 2-element subset of the set of colors assigned to the vertices of $G$. Since no two edges of $G$ are labeled the same, this vertex-coloring is edge-distinguishing. Since every vertex-coloring that assigns distinct colors to distinct vertices in a graph is a harmonious coloring, it follows that every graph has at least one harmonious coloring. The problem is to determine the minimum positive integer $k$ for which a graph $G$ has a harmonious coloring with $k$ colors. The harmonious coloring was introduced by Frank et al. [5] and independently by Hopcroft and Krishnamoorthy [8] in a slightly modified version, allowing that adjacent vertices may be colored with the same color.

Jendrol [9] generalized the harmonious coloring to multiset coloring. A (not necessarily proper) total-coloring $c$ (an assignment of colors to the vertices and the edges) of a graph $G$ is a multiset coloring if for any two edges $e_{1}=u_{1} v_{1}$ and 
$e_{2}=u_{2} v_{2}$ the associated triplets $\left\{c\left(u_{1}\right), c\left(e_{1}\right), c\left(v_{1}\right)\right\}$ and $\left\{c\left(u_{2}\right), c\left(e_{2}\right), c\left(v_{2}\right)\right\}$ are different.

Considering the edge-distinguishing property, we may restrict our attention to directed graphs. In this case we assign an ordered pair $(c(u), c(v))$ or an ordered triplet $(c(u), c(e), c(v))$ to every edge $e=u v$ of a directed graph and we can minimize the corresponding graph parameter. Budajová [2] introduced precisely this notion. Let $G=(V, E)$ be a directed graph with vertex set $V$ and edge set $E$. A mapping $c: V \cup E \rightarrow\{1, \ldots, k\}$ is called a total-coloring. A totalcoloring $c$ is edge-distinguishing if for any two edges $e_{1}=u_{1} v_{1}$ and $e_{2}=u_{2} v_{2}$ the associated ordered triplets $\left(c\left(u_{1}\right), c\left(e_{1}\right), c\left(v_{1}\right)\right)$ and $\left(c\left(u_{2}\right), c\left(e_{2}\right), c\left(v_{2}\right)\right)$ are different. The problem is to determine the minimum number of colors used in such a coloring of $G$. This parameter of $G$ is denoted by $\operatorname{hit}(G)$.

In this paper we investigate the parameter $h i t(G)$ for directed subdivided stars.

\section{Notation}

Let $G$ be a directed graph and let $P$ be a directed path in $G$. The length of $P$ is the number of its edges. A subdivision of $G$ is a directed graph resulting from a sequence of edge subdivisions applied to $G$. A subdivision of an edge $e=u v$ of $G$ is obtained by the addition of a new vertex $w$ into $G$ and replacement of $u v$ with two new edges $u w$ and $w v$.

The directed star $S_{d, 1}$ is a directed graph with vertex set $V=\left\{s_{0}, s_{1}, \ldots, s_{d}\right\}$ and edge set $E=\left\{s_{0} s_{j} ; j=1, \ldots, d\right\}$.

If we subdivide every edge of $S_{d, 1}$ exactly $r-1 \geq 0$ times, then we obtain the directed subdivided star $S_{d, r}$. The directed subdivided stars $S_{2,3}$ and $S_{4,2}$ are depicted in Figure 1.

Clearly, $S_{d, r}$ consists of $d$ directed paths of length $r$ which share one common vertex (the root of $S_{d, r}$ ). The $i$-th ray of $S_{d, r}$ is the directed path $s_{0}-s_{i}$.

\section{Results}

The exact values of $h i t\left(S_{d, r}\right)$ for sparse directed subdivided stars (directed subdivided stars with $\min \{d, r\} \leq 3$ ) were determined in [3, 4]. The following lemma gives a trivial lower bound for $\operatorname{hit}\left(S_{d, r}\right)$. 


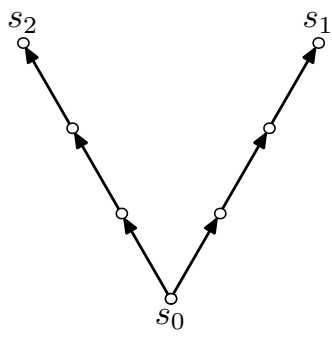

$S_{2,3}$

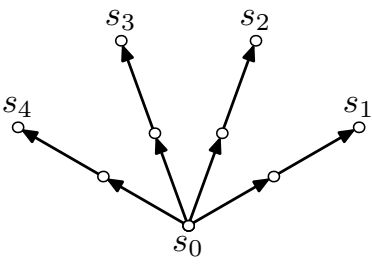

$S_{4,2}$

Figure 1: The directed subdivided stars $S_{2,3}$ and $S_{4,2}$.

Lemma 3. Let $d$ and $r$ be positive integers. Then

$$
\operatorname{hit}\left(S_{d, r}\right) \geq\lceil\sqrt[3]{d r}\rceil .
$$

Proof. We can create $k^{3}$ distinct ordered triplets using $k$ symbols. Therefore, if $G$ is a directed graph with $\operatorname{hit}(G)=k$, then it has at most $k^{3}$ edges. The directed graph $S_{d, r}$ has $d r$ edges, therefore $d r \leq\left(h i t\left(S_{d, r}\right)\right)^{3}$.

In this paper we prove that for sufficiently large $r$ it holds:

$$
\operatorname{hit}\left(S_{d, r}\right)=\lceil\sqrt[3]{d r}\rceil
$$

A complete directed graph is a directed graph in which every pair of distinct vertices is connected by a pair of unique edges (one in each direction). A directed cycle that passes through every vertex in a directed graph is called a directed Hamilton cycle. Alspach et al. [1] proved that every complete directed graph $K_{n}$ on $n \geq 2$ vertices admits an edge decomposition into directed Hamiltonian cycles except for $K_{4}$ and $K_{6}$.

Theorem 4. [1] Let $K_{n}$ denote the complete directed graph on $n$ vertices, $n \geq 2$. Then $K_{n}$ can be decomposed into directed cycles of length $n$ if and only if $n \notin\{4,6\}$.

A vertex-coloring $c$ of a directed graph $G$ is edge-distinguishing if for any two edges $u_{1} v_{1}$ and $u_{2} v_{2}$ of $G$ the associated ordered pairs $\left(c\left(u_{1}\right), c\left(v_{1}\right)\right)$ and $\left(c\left(u_{2}\right), c\left(v_{2}\right)\right)$ are different.

Recall that the union of two directed graphs $G_{1}=\left(V_{1}, E_{1}\right)$ and $G_{2}=$ $\left(V_{2}, E_{2}\right)$ with disjoint vertex sets $V_{1}$ and $V_{2}$ and edge sets $E_{1}$ and $E_{2}$ is the directed graph with vertex set $V_{1} \cup V_{2}$ and edge set $E_{1} \cup E_{2}$. We similarly define the union of $n$ directed graphs $G_{i}, 1 \leq i \leq n$, and we denote it $\bigcup_{i=1}^{n} G_{i}$. 
Lemma 5. Let $n \geq 2$ be a positive integer. Let $P_{i}=a_{i}-b_{i}$ be a directed path on $n$ edges, $i=1, \ldots, n-1$. Then there exists an edge-distinguishing proper vertex-coloring $c$ of $\bigcup_{i=1}^{n-1} P_{i}$ with colors $1, \ldots, n$ such that $c\left(a_{i}\right)=c\left(b_{i}\right)=n$ for $i=1, \ldots, n-1$. Moreover, if $n \notin\{4,6\}$, then each of the colors $1, \ldots, n$ appears on $P_{i}$ for $i=1, \ldots, n-1$.

Proof. First assume that $n \notin\{4,6\}$. Let $V\left(K_{n}\right)=\left\{u_{1}, \ldots, u_{n}\right\}$ be the vertex set of the complete directed graph $K_{n}$. Color the vertex $u_{i}$ with color $i$ for $i=1, \ldots, n$. By Theorem 4, the complete directed graph $K_{n}$ has an edge decomposition into $n-1$ directed cycles of length $n$. Let $C_{1}, \ldots, C_{n-1}$ be edgedisjoint directed cycles of length $n$ in $K_{n}$. If we split each of these cycles at $u_{n}$ (i.e. we replace $u_{n}$ by two vertices $u_{n_{1}}, u_{n_{2}}$ and replace the edges $x u_{n}, u_{n} y$ by edges $x u_{n_{1}}, u_{n_{2}} y$, respectively), then we obtain a proper vertex-coloring of $n-1$ paths on $n$ edges (the vertices $u_{n_{1}}$ and $u_{n_{2}}$ receive the color of $u_{n}$ ).

Now suppose (to the contrary) that the obtained vertex-coloring is not edge-distinguishing, i.e. there are two edges $g_{1} h_{1}$ and $g_{2} h_{2}$ such that $g_{1}$ has the same color as $g_{2}$ and $h_{1}$ has the same color as $h_{2}$. By the coloring of $K_{n}$, $g_{1}$ and $g_{2}$ correspond to the same vertex of $K_{n}$, say $u_{i}$, and similarly $h_{1}$ and $h_{2}$ correspond to the same vertex, say $u_{j}$. Consequently, $K_{n}$ contains two $u_{i} u_{j}$ edges, a contradiction.

If $n=4$, then we color the vertices of the directed paths $P_{1}, P_{2}, P_{3}$ with patterns: $4,1,2,1,4 ; 4,2,3,2,4$ and $4,3,1,3,4$.

Finally, if $n=6$, then we color the vertices of the five directed paths $P_{1}, P_{2}, P_{3}, P_{4}, P_{5}$ with the following patterns: $6,1,2,1,3,1,6 ; 6,2,3,2,4,2,6$; $6,3,4,3,5,3,6 ; 6,4,5,4,1,4,6$; and $6,5,1,5,2,5,6$.

Corollary 6. Let $n \geq 2$ be a positive integer. Let $P_{i}=a_{i}-b_{i}$ be a directed path on $n$ edges for $i=1, \ldots, n(n-1)$. Then $\bigcup_{i=1}^{n(n-1)} P_{i}$ admits an edge-distinguishing total-coloring with colors $1, \ldots, n$ such that adjacent vertices receive distinct colors, all vertices of degree one (i.e. vertices $a_{i}$ and $b_{i}$, $1 \leq i \leq n)$ receive color $n$, and the edge set of each $P_{i}$ is monochromatic.

Proof. Let $\mathcal{P}_{j}=\bigcup_{i=1}^{n-1} P_{i+(j-1)(n-1)}$ for $j=1, \ldots, n$. Observe that $\bigcup_{i=1}^{n(n-1)} P_{i}=$ $\bigcup_{j=1}^{n} \mathcal{P}_{j}$. By Lemma $5, \mathcal{P}_{j}$ admits an edge-distinguishing proper vertex-coloring $c$ with colors $1, \ldots, n$ such that all vertices of degree one receive color $n$. First we color the vertices of each $\mathcal{P}_{j}$ according to the coloring $c$. Thereafter we color 
all the edges in $\mathcal{P}_{j}$ with color $j$ for $j=1, \ldots, n$. In such a way we obtain a required total-coloring of $\bigcup_{j=1}^{n} \mathcal{P}_{j}=\bigcup_{i=1}^{n(n-1)} P_{i}$.

The main result of this paper is the following theorem.

Theorem 7. Let $d, r$ be positive integers and let $k=\lceil\sqrt[3]{d r}\rceil$. If $r \geq k^{2}$, then

$$
\operatorname{hit}\left(S_{d, r}\right)=\lceil\sqrt[3]{d r}\rceil
$$

Proof. If $k=1$, then necessarily $d=1$ and $r=1$. Evidently $\operatorname{hit}\left(S_{1,1}\right)=1$. So in the following we can assume that $k \geq 2$.

Let $r=x \cdot k+y$, where $x$ and $y$ are positive integers such that $1 \leq y \leq k$. Since $r \geq k^{2}$ we have $x \geq k-1$ (if $x<k-1$, then $x \cdot k<k^{2}-k$, consequently $\left.r=x \cdot k+y<k^{2}-k+k=k^{2}\right)$.

We will define an edge-distinguishing total-coloring of $S_{d, r}$ in three steps. In the first step we define an edge-distinguishing total-coloring of $S_{d, k(k-1)}$. In the second one we extend this coloring to an edge-distinguishing total-coloring of $S_{d, k(k-1)+y}$. Finally, in the third step, we define a required coloring of $S_{d, r}$.

By Corollary 6, there is an edge-distinguishing total-coloring of $k(k-1)$ directed paths of length $k$ with colors $1, \ldots, k$ such that no two adjacent vertices receive the same color, all endvertices of these paths are colored with color $k$, and the edge set of each path is monochromatic. Let $c$ be such a coloring of $k(k-1)$ directed paths of length $k$. The fact that $c$ is an edge-distinguishing total-coloring implies that there are exactly $k-1$ paths whose edges have the same color $i$; let $P_{i}^{1}, \ldots, P_{i}^{k-1}$ be these paths, $1 \leq i \leq k$.

Step 1: Since each directed path of length $k(k-1)$ is a concatenation of $k-1$ directed paths of length $k$, we can divide each ray of $S_{d, k(k-1)}$ into $k-1$ paths of length $k$. In this step we color the $k-1$ paths of length $k$ on the $i$-th ray, $1 \leq i \leq d$, in the same way as are colored $P_{i}^{1}, \ldots, P_{i}^{k-1}$ by the coloring $c$. This is always possible because from the fact $r \geq k^{2}$ it follows that $S_{d, r}$ has at most $k$ rays, i.e. $d \leq k$ (since otherwise, $d>k$ implies $d r>k^{3}$; consequently, $k=\lceil\sqrt[3]{d r}\rceil \geq \sqrt[3]{d r}>k)$

Step 2: By the coloring defined in Step 1, each ray of $S_{d, k(k-1)}$ contains a vertex of color $j$ for all $j \in\{1, \ldots, k\}$. Let $v^{j}$ be a vertex of color $j$ from the $j$-th ray, $j=1, \ldots, d$. In this step we replace the vertex $v^{j}$ from the $j$-th ray by a directed path of length $y$. Then we color the vertices and edges of this directed path with pattern $j, 1, j, 2, j, \ldots, j, y, j$ (each vertex receives color 
$j$ and the edges receive colors $1,2, \ldots, y)$. This is always possible since $y \leq k$. In such a way we obtain an edge-distinguishing total-coloring of $S_{d, k(k-1)+y}$.

Step 3: Now we extend this total-coloring of $S_{d, k(k-1)+y}$ to a required totalcoloring of $S_{d, r}$. Let $v_{0} v_{1}^{i} \ldots v_{r}^{i}$ denote the $i$-th ray of $S_{d, r}, i=1, \ldots, d$. The directed paths $v_{0} v_{1}^{i} \ldots v_{k(k-1)+y}^{i}, 1 \leq i \leq d$, are already colored; moreover, the endvertices of these paths are colored with $k$. Since the directed paths $v_{k(k-1)+y}^{i} \ldots v_{r}^{i}=v_{x k+y}^{i}$ have $(x k+y)-(k(k-1)+y)=k(x-(k-1))$ edges we can split them into directed paths of length $k$. We can color $(k-d)(k-1)$ of them according to the coloring $c$ of $P_{i}^{1}, \ldots, P_{i}^{k-1}$ for $d+1 \leq i \leq k$. Then we can "color" further $k-d$ such paths so that we replace a vertex of color $j, d+1 \leq j \leq$ $k$, by a directed path of length $k$ colored with pattern $j, 1, j, 2, j, \ldots, j, k, j$ (each vertex receives color $j$ and the edges receive colors $1, \ldots, k$ ). If all the elements of $S_{d, r}$ are colored, then we are ready. In the other case every uncolored edge $e=u v$ must receive one of the triplets $(j, \ell, j)$ with $y+1 \leq \ell \leq k$ and $1 \leq j \leq d$ since all other triplets are already used. We "color" the uncolored elements step by step, we always replace a vertex of color $j$ by a path of length 1 colored with pattern $j, \ell, j$. In such a way we can use all $k^{3}$ triplets. Since $S_{d, r}$ has no more than $k^{3}$ edges $\left(k=\lceil\sqrt[3]{d r}\rceil \geq \sqrt[3]{d r}\right.$, consequently $\left.k^{3} \geq d r=\left|E\left(S_{d, r}\right)\right|\right)$ the proof is complete.

\section{Acknowledgments}

This work was supported by the Slovak Research and Development Agency under the Contract No. APVV-15-0116.

\section{References}

[1] B. Alspach, H. Gavlas, M. Šajna, H. Verrall, Cycle decomposition IV: complete graphs and fixed length directed cycles, J. Combin. Theory Ser. A, 103 (2003), 165-208, doi: 10.1016/S0097-3165(03)00098-0.

[2] K. Budajová, Total edge irregular number of digraphs, Acta Avionica, 21 (2011), 12-16.

[3] K. Budajová, J. Czap, Hit number of directed bipartite graphs, Appl. Math. Sciences, 6 (2012), 5689-5693.

[4] K. Budajová, J. Czap, Hit number of sparse subdivided stars, Appl. Math. Sciences, 6 (2012), 6753-6759.

[5] O. Frank, F. Harary, M. Plantholt, The line-distinguishing chromatic number of a graph, Ars Combin., 14 (1982), 241-252. 
[6] S.W. Golomb, How to number a graph, Graph Theory and Computing, Academic Press, New York (1972), 23-37.

[7] R.L. Graham, N.J.A. Sloane, On additive bases and harmonious graphs, SIAM J. Alg. Disc. Meth., 1 (1980), 382-404, doi: 10.1137/0601045.

[8] J. Hopcroft, M.S. Krishnamoorthy, On the harmonious coloring of graphs, SIAM J. Alg. Disc. Meth., 4 (1983), 306-311, doi: 10.1137/0604032.

[9] B. Jendrol̆, Total edge-distinguishing number, Doctoral thesis, University of P.J. Šafárik (2002) (in Slovak).

[10] A. Rosa, On certain valuations of the vertices of a graph, Theory of Graphs, Gordon and Breach, New York (1967), 349-355. 